\title{
The combined effect of parathyroid hormone (1-34) and whole-body vibration exercise on physical performance in OSteoporotic women (PaVOS study): a secondary analysis from a randomised controlled trial
}

Ditte Beck Jepsen ${ }^{1,2^{*}}$ (D), Tahir Masud ${ }^{1,2,3}$, Anders Holsgaard-Larsen ${ }^{2,4}$, Stinus Hansen ${ }^{5,6}$, Niklas Rye Jørgensen ${ }^{7,8}$ and Jesper Ryg ${ }^{1,2}$

\begin{abstract}
Background: The aim of this study was to investigate the effect on physical performance of combining wholebody vibration exercise (WBV) with parathyroid hormone 1-34 (teriparatide) compared to teriparatide alone.

Methods: A secondary analysis from a RCT where postmenopausal women with severe osteoporosis were randomised to WBV plus teriparatide (intervention) or teriparatide alone (control). WBV was applied three times/week (6x1min WBV: 1 min rest, (peak acceleration $3.6 \mathrm{~g}$ )) for twelve months. Both groups received teriparatide $20 \mu \mathrm{g} \mathrm{s.c./day.} \mathrm{The} \mathrm{primary}$ endpoint (bone mineral density) is reported elsewhere. Physical performance measures (Short Physical Performance Battery (SPPB), Timed-Up-and-Go (TUG), leg extension power, and grip strength) were obtained at baseline, three-, six-, and twelve months, lean mass at baseline and twelve months. Data were analysed with mixed linear regression model or robust cluster regression in an intention to treat analysis.
\end{abstract}

Results: Thirty-five women aged (mean \pm SD) $69 \pm 7$ ) years were recruited of which thirty-two (91\%) completed the twelve months follow-up (WBV + teriparatide $=15$, teriparatide $=17)$. SPPB score (mean \pm SD) improved significantly at three months in the WBV + teriparatide group from $9.13 \pm 2.03$ to $10.35 \pm 1.69(p=0.014)$ with a statistical trend towards a between-group change in favor of the WBV + teriparatide group $(0.86[95 \% \mathrm{Cl}(-0.05,1.77), p=0.065])$. Both groups improved in leg extension power during the study period whereas no changes were seen in TUG, grip strength, or lean mass in either group. No statistical significant between-group differences were observed.

(Continued on next page)

\footnotetext{
* Correspondence: Ditte.Beck.Jepsen@rsyd.dk

'Department of Geriatric Medicine, Odense University Hospital, Odense, Denmark

${ }^{2}$ Department of Clinical Research, University of Southern Denmark, Odense, Denmark

Full list of author information is available at the end of the article
}

(c) The Author(s). 2020 Open Access This article is licensed under a Creative Commons Attribution 4.0 International License, which permits use, sharing, adaptation, distribution and reproduction in any medium or format, as long as you give appropriate credit to the original author(s) and the source, provide a link to the Creative Commons licence, and indicate if changes were made. The images or other third party material in this article are included in the article's Creative Commons licence, unless indicated otherwise in a credit line to the material. If material is not included in the article's Creative Commons licence and your intended use is not permitted by statutory regulation or exceeds the permitted use, you will need to obtain permission directly from the copyright holder. To view a copy of this licence, visit http://creativecommons.org/licenses/by/4.0/. The Creative Commons Public Domain Dedication waiver (http://creativecommons.org/publicdomain/zero/1.0/) applies to the data made available in this article, unless otherwise stated in a credit line to the data. 
(Continued from previous page)

Conclusion: WBV may improve some short-term aspects of physical performance in severely osteoporotic postmenopausal women who are receiving teriparatide treatment.

Trial Registration: ClinicalTrials.gov, ID:NCT02563353

Keywords: Exercise, Osteoporosis, Physical performance, Short physical performance battery, Whole-body vibration, Women

\section{Background}

Osteoporosis and increased propensity to fall are major determinants of fragility fractures in an aging population. The economic burden of such fractures is high [1] and expected to rise with increasing life expectancy [2]. Moreover, the individual burden of a fragility fracture is substantial with decreased quality of life, risk of dependence, and increased mortality [3, 4]. Several pharmaceutical agents are approved for the treatment of osteoporosis and have been shown to reduce the risk of fragility fractures $[5,6]$. The anabolic agent parathyroid hormone analogue 1-34 (teriparatide) is capable of increasing bone mineral density (BMD) in lumbar spine and proximal femur in postmenopausal women with osteoporosis [7]. The risk of falling is multifactorial with decreased neuromuscular function and impaired balance being prominent risk factors [8]. Exercise programs that challenge balance can reduce fall rates in community-dwelling older adults and those with higher training intensity yielding greater effects [9]. Whole-body vibration exercise (WBV) has emerged as an alternative training intervention that is well tolerated in women with osteoporosis and even in older adults with a high risk of falls $[10,11]$. WBV has the capacity to improve muscle strength of the lower extremities and body balance in older adults, both important factors in decreasing the risk of falls [12-14]. A previous study has shown a positive effect of combining osteoporotic treatment i.e. alendronate and exercise, where alendronate increased bone mass, exercise improved the participants' muscular performance and dynamic balance, potentially decreasing the risk of fractures [15].

We have previously shown that combining pharmacological treatment for osteoporosis in the form of teriparatide with WBV as an exercise increased BMD of lumbar spine by an additional $2.95 \%$ [16]. If in addition WBV also can increase physical performance, then this could potentially result in an optimized fracture prevention intervention. However, the potential combined effect of this dual approach on physical performance is unknown.

Therefore, the aim of this pre-defined secondary analysis from a 12 month randomized controlled trial (RCT) was to investigate the effect on physical performance of combining WBV with teriparatide treatment compared to teriparatide alone in postmenopausal women with severe osteoporosis.

\section{Methods}

This study is a sequel analysis of a RCT [17]. Outcome measures for the present analysis were a priori defined in a statistical analysis plan at ClinicalTrials.gov (NCT02563353) and in the published study protocol [17]. Data collection for this study started November 2015 and was finalized November 2018 and study results are reported according to the CONSORT statement [18].

\section{Participants}

The recruitment of women starting teriparatide was performed in five outpatient clinics: the Osteoporosis Clinic or Department of Geriatric Medicine (Odense University Hospital), Department of Endocrinology (Hospital South West Jutland), Department of Endocrinology (Hospital Lillebaelt), or Department of Endocrinology and Internal Medicine (Aarhus University Hospital). Inclusion criteria were: postmenopausal women $\geq 50$ years of age, with either one vertebral fracture within the last 3 years with $>25 \%$ reduction in vertebral height and BMD at the lumbar spine or total hip with T-score $<-3$, or at least two vertebral fractures with $>25 \%$ reduction in vertebral height, with no requirements for low BMD, planned to start treatment with teriparatide. Exclusion criteria were: ongoing oral glucocorticoid treatment, inability to tolerate WBV for $1 \mathrm{~min}$ at screening, not being able to give informed consent, or contraindications to WBV i.e. pacemakers and knee or hip joint replacements.

\section{Design and randomization}

A 12 month, superiority- RCT with two parallel arms investigating the effect of combined WBV and teriparatide compared to teriparatide alone was conducted from November 2015 to November 2018. The investigator who performed the web-randomization was not involved in outcome assessment. Sham WBV was not utilized, but the assessors performing the outcome measurements were blinded to group assignment. A more detailed description can be found elsewhere in the published study protocol [17].

All participants received subcutaneous teriparatide $20 \mu \mathrm{g} /$ day. Web-based block randomization was conducted at baseline after eligibility criteria were satisfied and the baseline measurements were obtained. 


\section{Intervention and adherence}

The WBV was performed on a Power plate My5 (Power plate $\left.^{\circ}, \mathrm{UK}\right)$, using a protocol that was well tolerated in a previous study in frail older individuals with a frequency of $30 \mathrm{~Hz}$ and amplitude of $1 \mathrm{~mm}$ (low displacement) with peak acceleration of $35.53 \mathrm{~m} \mathrm{~s}^{-2} \mathrm{rms}(3.6 \mathrm{~g})$ [10]. The training was conducted at the participants own homes and consisted of up to 12 min of training with the WBV:rest ratio 1:1 min, including 6 min of vibration, 3 days a week with 1 day pause in between. The training progressed the first 8 weeks up to the 6 min of vibration to ensure that participants could tolerate the training in the total 12 months. The training protocol followed the safety ISO-guidelines [19] and has previously been shown to be safe in a population of older adults aged 80 years in term of falls risk [10]. All participants trained without shoes. They were instructed at home and performed their first training supervised. The participants were followed with monthly telephone calls and were instructed to contact the project manager if they needed clarification or had questions. Where necessary the instructor re-visited the participants to ensure that the training was conducted as instructed. The protocol and monitoring by attendance log has previously been described elsewhere [17]. High adherence to WBV was defined as being $>75 \%$ adherent (i.e. more than 685 exercise sessions out of 913). Falls were assessed through calendars and monthly telephone contact was used to ensure adherence and to collect data on pain, dizziness, falls, and other adverse events. Physical activity was assessed using the international physical activity questionnaire (IPAQ) [20].

\section{Outcomes}

The primary outcome of BMD is reported elsewhere [16]. This study reports predefined secondary outcomes of physical performance, muscle function, and lean mass measures.

Included measures were: Short Physical Performance Battery (SPPB) [21], Timed-Up-and-Go test (TUG), leg extensor power [22], and hand grip strength [23]. SPPB consists of a five times sit to stand test (5TSTS), a three meter walking test and a tandem test. SPPB was scored according to guidelines and calculated using the points score validated to assess lower extremity function and balance in older adults [21]. The time used to perform the TUG was recorded for the participants to rise from a chair, walk three meters, return and sit back down, with three repetitions. Unilateral leg extension power was assessed using a Nottingham leg extensor power rig, (Medical Engineering Unit, School of Biomedical Sciences, University of Nottingham Medical School, Nottingham, UK) measuring peak extension power [22]. Hand grip strength was measured using a Smedley dynamometer (TTM; Tokyo, Japan). The measurements were done according to standard protocol, using the peak values from the bilateral testing [23].

The physical tests were performed at baseline, three-, six-, and 12 months of follow-up by trained technicians using a standardized protocol. With repeated measurements the participants' best test score was applied. Reported coefficients of variation for TUG, 5TSTS, and leg power were $5.3,5.1$, and $9 \%$, respectively $[22,24]$.

Whole body dual-energy X-ray absorptiometry (DXA) (Hologic Discovery, Waltham, MA, USA) was performed to assess muscle mass at baseline and 12 months. Appendicular lean mass was determined using the equation: appendicular lean mass $(\mathrm{kg}) /$ height $(\mathrm{m})^{2}$.

\section{Statistical methods}

The sample size was calculated from expected changes in the primary outcome of BMD [17]. Allowing for $20 \%$ drop out rate the inclusion of 40 participants was planned. Power and sample size considerations for this follow-up study on secondary outcomes were evaluated a posteriori.

Data were presented with means and standard deviations or medians and interquartile ranges depending on distribution. Between-group differences in baseline characteristics and study adverse events were assessed using the Student t-test, Wilcoxon rank sum, or the chi-square test according to continues outcomes with normal or non-normal distributions, or categorical outcomes respectively. The treatment groups were compared using a linear mixed-effect model with fixed effects for treatment groups at baseline, time, and time by treatmentgroup interaction. Data that had non-normal distributed residuals were analyzed in a linear regression model with robust cluster estimation with clusters to deal with the individuals' effect on the repeated measurements. Model assumptions were checked using histograms, plots of the residuals against fitted values, and normal probability plots of the residuals. The primary mixed multilevel model included all observed values, whereas a secondary analysis applied multiple imputations to missing values. A significance level of 0.05 was chosen. All statistical analyzes were performed using the Statistical Package STATA (version 14 and 15, Stata Corp., College Station, TX, USA). Intention to treat (ITT) and per protocol (PP) analysis were conducted to investigate if adherence (> 75\%) affected the results.

\section{Results}

The recruitment centers informed 93 eligible women who were starting teriparatide treatment about the study. A total of 35 postmenopausal women aged (mean \pm SD) $69 \pm 7$ (range 53-81) years were included (Fig. 1). Of the 35 included participants 33 (94\%) 


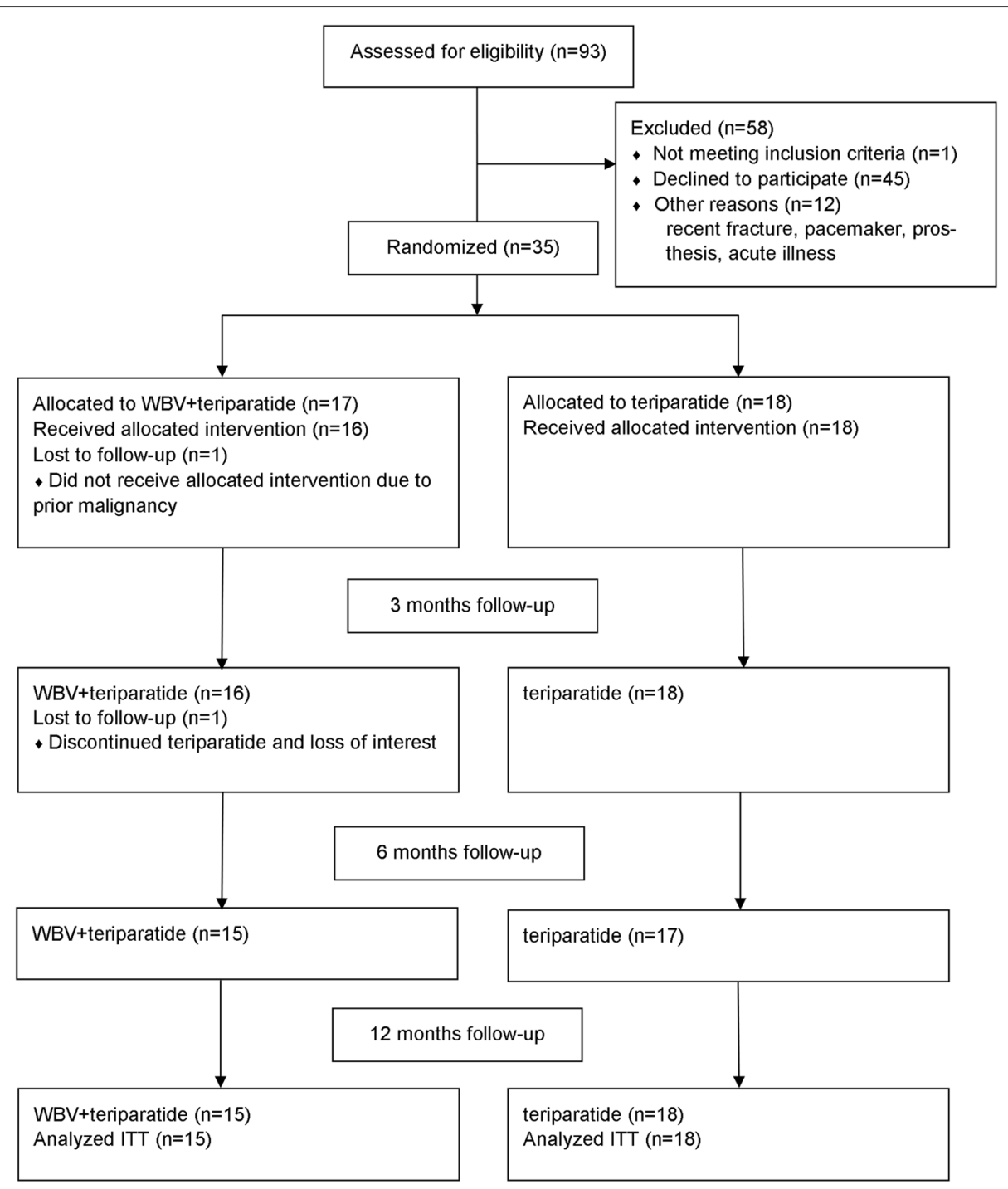

Fig. 1 Consort flow diagram showing number of subjects screened for study participation and number of participants in each treatment group at baseline, three-, six-, and twelve months of follow-up, with designation of reasons for early discontinuation. The WBV + teriparatide group received whole-body vibration and teriparatide and the teriparatide group received teriparatide alone. WBV whole-body vibration, ITT intention to treat

completed 3 months-, 32 (91\%) completed 6 months-, and 32 (91\%) completed 12 months examinations. In the $\mathrm{WBV}+$ teriparatide group, one participant never started teriparatide and was lost to follow-up after baseline measurements, one participant was lost to follow-up after 3 months, one participant did not attend 3 months followup but attended six- and 12 months follow-up, and two participants discontinued the WBV after 3 months but continued teriparatide and attended the follow-up visits. In the teriparatide group, one participant was lost to follow-up after 3 months. The consort flow diagram illustrates recruitment and participants' flow through the study (Fig. 1). Baseline characteristics of the study population are shown in Table 1. There were no significant differences between the two groups.

\section{Physical performance and muscle function}

The observed physical performance measurements are presented in Table 2.

The WBV + teriparatide group improved significantly in SPPB (mean \pm SD) at 3 months compared to baseline from $9.13 \pm 1.96$ to $10.35 \pm 1.69 \quad(p=0.014)$ (mainly driven by an improvement in 5TSTS) (Table 2), whereas no significant change was seen in the teriparatide group $(10.16 \pm 1.82$ vs $10.17 \pm 2.00)$. A trend towards a difference between-group in favor of the WBV + teriparatide group was observed for SPPB score at 3 months 0.86 [95\%CI $(-0.05,1.77), p=0.065]$. Also, a post hoc analysis (including participants using their arms) showed a statistical significant between-group change in favor of the WBV + teriparatide group for 5TSTS score at 12 months 
Table 1 Baseline characteristics of study participants treated with whole-body vibration and teriparatide compared to teriparatide alone

\begin{tabular}{|c|c|c|c|}
\hline & WBV + teriparatide group $(n=17)$ & teriparatide group $(n=18)$ & $P$ value \\
\hline Age (years) (mean $\pm S D)$ & $69 \pm 5$ & $69 \pm 8$ & 0.909 \\
\hline $\mathrm{BMI}\left(\mathrm{kg} / \mathrm{m}^{2}\right)($ mean $\pm \mathrm{SD})$ & $24.5 \pm 4.1$ & $24.1 \pm 4.3$ & 0.797 \\
\hline \multicolumn{4}{|l|}{ Charlson comorbidity score, median (IQR) } \\
\hline Smoking, n (\%) & $1(0-1)$ & $0(0-0)$ & 0.087 \\
\hline Current & $4(24)$ & $4(22)$ & 0.630 \\
\hline Former & $6(35)$ & $9(50)$ & \\
\hline Never & $7(41)$ & $5(28)$ & \\
\hline \multicolumn{4}{|l|}{ Physical activity at baseline*, n (\%) } \\
\hline Low & $10(59)$ & $8(39)$ & 0.421 \\
\hline moderate & $4(23)$ & $8(50)$ & \\
\hline High & $3(18)$ & $2(11)$ & \\
\hline \multicolumn{4}{|l|}{ Falls in the last 12 monthsa, n (\%) } \\
\hline None & $10(59)$ & $11(61)$ & 0.244 \\
\hline 1 & $3(18)$ & $6(33)$ & \\
\hline$\geq 2$ & $4(24)$ & $1(6)$ & \\
\hline \multicolumn{4}{|l|}{ Alcohol, (units a day)a, n (\%) } \\
\hline none & $3(18)$ & $6(33)$ & 0.543 \\
\hline up to 1 & $11(64)$ & $10(56)$ & \\
\hline$>1$ & $3(18)$ & $2(11)$ & \\
\hline Ability to rise from chair without using arms $x, \mathrm{n}(\%)$ & $12(71)$ & $16(89)$ & 0.177 \\
\hline
\end{tabular}

$P$ values for difference t-test when normal distribution, chi squared for categorical, mann-whitney test for continual non-normal distribution. ${ }^{*}$ Estimated using the IPAQ questionnaire, self-reported outcome. a Self-reported outcome BMI body mass index, WBV whole-body vibration

-4.7 s $[95 \%$ CI $(-9.1,-0.3), p=0.037]$. The WBV + teriparatide group had a significantly higher 5TSTS time at baseline compared to the teriparatide group which was adjusted for in the linear regression model. The WBV + teriparatide group improved significantly in 5TSTS (mean $\pm \mathrm{SD}$ ) at three and 12 months of follow-up $(18.9 \pm 7.9 \mathrm{~s}, 14.4 \pm 6.9 \mathrm{~s}$, and $15.7 \pm 9.8 \mathrm{~s}$ at baseline, 3 months, and 12 months, respectively $(p<0.05))$. A sensitivity analysis excluding participants who used their arms for support revealed a between-group change score of $-7.3 \mathrm{~s}$ [95\%CI $(-11.7,-2.8), p=0.002$ ] at 12 months with a nonsignificant improvement in the WBV + teriparatide group at three- and 6 months and a significant improvement at 12 months (compared to no significant improvements in the teriparatide group). There were no significant difference between the groups in the time for walking three meters (mean $\pm \mathrm{SD}$ ); however, it increased significantly in the WBV + teriparatide group at 12 months $(2.9 \pm 0.7$ at baseline vs $3.4 \pm 1.8$ at 12 months, $p=0.049$ ) (Table 2). Both groups improved significantly in leg extension power during the study with no significant difference between groups (Table 2). No significant change in hand grip strength, TUG, total lean mass, or appendicular lean mass was observed at any time point in either group (Table 2). No statistical between-group differences were observed.

When missing data were replaced by multiple imputations the results were similar to those observed with the within-group improvement in SPPB in the WBV + teriparatide group at 3 months being +0.89 [95\%CI $(0.06,-1.71)$, $p=0.035]$; however, the between-group change in SPPB at 3 months were 0.89 [95\%CI $(-0.19,1.97), p=0.107]$.

Overall, the PP analysis demonstrated similar results as the ITT, and no changes between groups were statistically significant (data not shown). However, the PP analysis did show a significant improvement in TUG (mean $\pm \mathrm{SD}$ ) in the WBV + teriparatide group from $10.7 \pm 3.7 \mathrm{~s}$ at baseline to $9.2 \pm 2.8 \mathrm{~s}$ at 3 months ( $p=$ 0.045 for change within-group with no statistical significant difference between groups). There were no significant between group changes in self-reported physical activity (mean MET/min) from baseline to 12 months follow-up. However, both groups increased their activity levels $(p<0.05)$ (WBV + teriaparatide group: low $=59 \%$, moderate $=23 \%$, and high $=18 \%$ changed to low $=33 \%$, moderate $=27 \%$, and high $=40 \%$ ) (Teriaparatide group: low $=39 \%$, moderate $=40 \%$, and high $=11 \%$ changed to low $=13 \%$, moderate $=63 \%$, and high $=25 \%$ ). 
Table 2 Physical performance measures at baseline, three, six, and twelve months of follow-up in patients treated with whole-body vibration and teriparatide (intervention) compared to teriparatide alone (control)

\begin{tabular}{|c|c|c|c|c|c|}
\hline & Intervention & $\begin{array}{l}\text { P change within } \\
\text { group from baseline }\end{array}$ & Control & $\begin{array}{l}\text { P change within } \\
\text { group from baseline }\end{array}$ & $\begin{array}{l}\text { P change between } \\
\text { groups from baseline }\end{array}$ \\
\hline SPPB, point & Mean \pm sd & & Mean \pm sd & & \\
\hline Baseline & $9.13 \pm 1.96 n=16$ & & $10.16 \pm 1.82 n=18$ & & \\
\hline Three months & $10.35 \pm 1.69 n=14$ & $0.014^{*}$ & $10.17 \pm 2.00 n=18$ & 1.000 & 0.065 \\
\hline Six months & $9.46 \pm 2.29 n=15$ & 0.128 & $10.17 \pm 1.70 n=17$ & 0.742 & 0.372 \\
\hline Twelve months & $9.57 \pm 2.50 n=14$ & $0.043^{*}$ & $10.24 \pm 1.82 n=17$ & 0.606 & 0.248 \\
\hline \multicolumn{6}{|c|}{$5 \times$ Chairstand time, with arms, s } \\
\hline Baseline & $18.9 \pm 7.9 n=16$ & & $13.8 \pm 4.6 n=18$ & & \\
\hline Three months & $14.4 \pm 6.9 n=14$ & $0.036^{*}$ & $13.0 \pm 3.5 n=18$ & 0.395 & 0.117 \\
\hline Six months & $16.2 \pm 6.3 n=15$ & 0.085 & $13.4 \pm 3.7 n=17$ & 0.572 & 0.185 \\
\hline Twelve months & $15.7 \pm 9.8 n=14$ & $0.040^{*}$ & $15.3 \pm 6.6 n=17$ & 0.341 & 0.037 \\
\hline \multicolumn{6}{|l|}{ Time $3 \mathrm{~m}$ walk, s } \\
\hline Baseline & $2.9 \pm 0.7 n=17$ & & $2.8 \pm 0.9 n=18$ & & \\
\hline Three months & $2.7 \pm 0.5 n=15$ & 0.722 & $2.8 \pm 0.8 n=18$ & 0.848 & 0.896 \\
\hline Six months & $3.0 \pm 1.0 n=15$ & 0.977 & $2.8 \pm 0.9 n=17$ & 0.912 & 0.956 \\
\hline Twelve months & $3.4 \pm 1.8 n=15$ & $0.049^{*}$ & $2.7 \pm 0.8 n=17$ & 0.622 & 0.077 \\
\hline \multicolumn{6}{|l|}{ Tandem total, s } \\
\hline Baseline & $27.8 \pm 4.6 n=17$ & & $28.8 \pm 2.8 n=18$ & & \\
\hline Three months & $28.3 \pm 2.5 n=15$ & 0.525 & $28.4 \pm 3.0 n=18$ & 0.680 & 0.470 \\
\hline Six months & $28.8 \pm 2.4 n=15$ & 0.400 & $27.7 \pm 3.1 n=17$ & 0.255 & 0.177 \\
\hline Twelve months & $28.9 \pm 2.3 n=15$ & 0.191 & $28.3 \pm 2.9 n=17$ & 0.226 & 0.094 \\
\hline \multicolumn{6}{|l|}{ TUG, s } \\
\hline Baseline & $10.7 \pm 3.2 n=17$ & & $9.6 \pm 3.1 n=18$ & & \\
\hline Three months & $9.2 \pm 2.5 n=15$ & 0.257 & $9.2 \pm 2.8 n=18$ & 0.580 & 0.650 \\
\hline Six months & $10.0 \pm 4.3 n=15$ & 0.395 & $9.3 \pm 4.3 n=17$ & 0.668 & 0.746 \\
\hline Twelve months & $11.4 \pm 7.2 n=15$ & 0.394 & $8.95 \pm 2.9 n=17$ & 0.344 & 0.205 \\
\hline \multicolumn{6}{|c|}{ Leg extension power, W/kg } \\
\hline Baseline & $0.91 \pm 0.37 n=17$ & & $1.10 \pm 0.43 n=18$ & & \\
\hline Three months & $1.07 \pm 0.42 n=15$ & 0.175 & $1.24 \pm 0.48 n=18$ & $0.026^{*}$ & 0.606 \\
\hline Six months & $1.13 \pm 0.50 n=15$ & $0.010^{*}$ & $1.27 \pm 0.64 n=17$ & $0.017^{*}$ & 0.798 \\
\hline Twelve months & $1.08 \pm 0.49 n=15$ & $0.044^{*}$ & $1.36 \pm 0.53 n=17$ & $0.000^{*}$ & 0.339 \\
\hline \multicolumn{6}{|l|}{ Total Lean mass, kg } \\
\hline Baseline & $38.53 \pm 6.56 n=17$ & & $37.04 \pm 3.88 n=18$ & & \\
\hline Twelve months & $38.20 \pm 6.65 n=15$ & 0.281 & $37.04 \pm 4.84 n=17$ & 0.657 & 0.276 \\
\hline \multicolumn{6}{|c|}{ Appendicular lean mass index $\mathrm{kg} / \mathrm{m}^{2}$} \\
\hline Baseline & $5.89 \pm 0.76 n=17$ & & $6.10 \pm 0.89 n=18$ & & \\
\hline Twelve months & $5.84 \pm 0.66 n=15$ & 0.421 & $6.13 \pm 0.92 n=17$ & 0.692 & 0.391 \\
\hline
\end{tabular}

$P$ values are derived from a mixed linear regression model with time, time $x$ treatment interaction and adjustment for baseline group values, or robust cluster regression model with time, time $x$ treatment and adjustment for baseline group values with clusters for repeated measurements. ${ }^{*}$ Indicates a significant change $p<0,05 . \mathrm{n}=$ number participants, SPPB short physical performance battery, TUG timed up and go

\section{Adherence and adverse events}

A total of $13(76.5 \%)$ participants reported $>75 \%$ WBV training adherence (i.e. more than 685 sessions out of 913). Two participants reported pain in the lower extremities and one of them stopped the WBV prematurely without any reasons related to the WBV.

A total of 33 participants (94\%) had adherence $>80 \%$ with the teriparatide treatment based on the percentage 
of collected prescribed prescriptions with no significant differences between groups. Also, no significant change in reported pain or dizziness was seen within- or between-groups. A few serious adverse events were reported: five hospital admissions (four in the teriparatide group and one in the WBV + teriparatide group), one distal femur fracture (WBV + teriparatide group), and one vertebral fracture (WBV + teriparatide group). No serious adverse events were believed to be related to WBV. In the 12 months of follow-up, two participants in the teriparatide group reported one fall each. In the $\mathrm{WBV}+$ teriparatide group, three participants fell once, one participant fell twice, and one participant fell three times. No falls occurred in relation to the training. There were no significant differences in fallers, falls, or adverse events between the two groups.

\section{Discussion}

In this study that reports pre-specified secondary outcomes from a 12 months RCT comparing WBV and teriparatide with teriparatide alone in osteoporotic women some beneficial effects of WBV + teriparatide on physical performance were seen. The minimal clinically important change estimates for SPPB in older adults is $0.3-0.8$ points and improvements of $0.4-1.5$ points are associated with a substantial change in self-reported mobility [25]. The WBV + teriparatide group in our study had clinical relevant improvements in SPPB (0.86), mainly driven by improvements in the 5TSTS. Leg extension power improved statistically in both groups whereas no clinical or statistical improvements were observed in TUG, grip strength, or muscle mass.

While no prior studies have addressed the combined effect of WBV and teriparatide others have reported mixed results regarding the effects of WBV on physical parameters in postmenopausal women. One study found a positive effect of WBV on jumping height in postmenopausal women with osteoporosis compared to combined balance and resistance training [12], while a recent study did not find an effect of WBV on leg extension power or jumping performance in osteopenic postmenopausal women [26]. Another study using the same WBV training protocol as used in our study did however find a positive effect of WBV on leg extension power in older adults [10]. The specific WBV training protocol may be important because differences in duration, repetitions, vibration frequencies, and amplitude result in different training acceleration and training dosage. The improvements in SPPB in the WBV + teriparatide group in our trial were at its highest at 3 months of treatment but this effect was not fully sustained throughout the study. This might be due to the progression period of the training program in the first 2 months where the number of training sessions increased or due to a lower persistence with time. There was a trend towards a treatment and time interaction at 3 months; however, the trial had insufficient power to detect significant effects between groups. Indeed, a posteriori power and sample size evaluation for this follow-up study on secondary outcomes showed that a total of 64 participants (32 in each group) would have been needed to complete the trial in order to yield sufficient power $(>80 \%)$ to detect a between-group difference $(p<0.05)$ of the observed 0.86 SPPB points in the multilevel linear mixed regression analysis. The PP analysis showed similar results as the ITT but with a smaller sample size and no changes between groups were significant. The PP revealed a significant improvement in TUG in the WBV + teriparatide group at 3 months of follow-up although this effect was not sustained.

In our study both groups improved in the observed leg extension power, which could be due to clinical reasoning together with a potential learning effect of the test procedure or that the participants in the teriparatide group participated in physical activity not related to the study. We assessed the participants' physical activity levels at baseline and 12 months of follow-up via the IPAQ questionnaire and found a significant increase in physical activity levels in both groups at 12 months with no significant difference in the changes between groups. Physical activity not related to the study could affect our results but it would be unethical to limit habitual physical activity in the teriparatide group in light of the recommendations of the importance of exercise in this population [27].

Our study was not powered nor aimed at investigating a combined effect of WBV and teriparatide on falls. However, the participants in the $\mathrm{WBV}+$ teriparatide group experienced more falls during the study period compared to the teriparatide alone group, and this might be explained partly by a higher comorbidity index in the former group $(p=0.087)$. Furthermore, the $\mathrm{WBV}+$ teriparatide group performed significantly worse in the 5TSTS at baseline (18.9 $\mathrm{s}$ versus $13.8 \mathrm{~s}$ in the $\mathrm{WBV}+$ teriparatide and teriparatide alone group, respectively). A 5TSTS test above $15 \mathrm{~s}$ has previously been shown to be a predictor of recurrent falls $[28,29]$. This may have affected the results due to the former group being at higher risk of falls at baseline compared to the latter group. To study the effect on falls per se it would require a much larger trial. Nevertheless a recent metaanalysis showed that WBV can reduce fall rates in line with other fall preventive training programs $[30,31]$.

\section{Study limitations}

Some limitations of our study design and protocol should be noted. The participants were not blinded to the WBV intervention; however, the assessors obtaining 
the physical measurements were blinded to group allocation. Furthermore, the training was conducted unsupervised at the participants' own homes and we do not know if the participants followed the instructions even though monthly telephone contact was made. The "at home" training design was chosen to ensure high adherence and availability since known barriers to exercise are transportation and lack of time [32]. Adherence was assessed through training logbooks and this method has previously been reported to be reliable with an excellent overall intra-class correlation of 0.96 compared to electronic monitoring [33]. Finally, the sample size had low power to detect changes in the secondary outcomes of physical performance measures. Although we were able to detect a clinically relevant improvement in SPPB, no significant difference between groups were detected and our results should therefore be interpreted with caution.

The study was conducted according to guidelines, registered at the online registry clinicaltrials.gov, and the protocol was published with high internal validity in the study conduct. Of the informed eligible patients starting teriparatide approximately one out of three were included in the study. The included participants might differ from the overall patient population treated with teriparatide; however, age and comorbidity are comparable to patients from a large register study assessing all patients treated with teriparatide in during 2003-2010 [34] giving our study high external validity. However, we only included women in our study and the results may therefore not be generalizable to men. The results of this study highlight the feasibility of WBV training in osteoporotic women with a high attendance rate of $91 \%$ completing 12 months of follow-up. Future trials could investigate the effect of WBV in a fall preventive setting, combining treatment of osteoporosis and WBV to improve physical performance in a dual approach to lower the risk of fragility fracture.

\section{Conclusion}

In conclusion, the results of the current study show that combining WBV with teriparatide in post-menopausal women with severe osteoporosis is feasible and capable of improving some aspects of physical performance. The clinically relevant improvements in SPPB were seen after 3 months of training but were not fully sustained for the remainder of the trial. Whether increasing WBV intensity and progression would lead to improved mobility and reduced falls risk needs further investigation.

\section{Abbreviations}

5TSTS: Five times sit to stand; BMD: Bone mineral density; DXA: Dual-energy X-ray absorptiometry; IPAQ: International physical activity questionnaire; ITT: Intention to treat; PP: Per protocol; RCT: Randomized controlled trial; SPPB: Short physical performance battery; TUG: Timed-up-and-go; WBV: Whole-body vibration exercise

\section{Acknowledgments}

We would like to thank all participants and the inclusion centres. We are grateful to Mrs. Steffanie Anthony Christiansen and Mrs. Jane Nielsen for assistance with study coordination.

\section{Authors' contributions}

Study design: TM, DJ, and JR. Study conduct: DJ, TM, and JR. Data collection: DJ. Data analysis: DJ, TM, and JR. Data interpretation: DJ, TM, AL, SH, NRJ, and JR. Drafting the manuscript: DJ. Revising manuscript: DJ, TM, AL, SH, NRJ, and $J R$. Approving final version of manuscript: DJ, TM, AL, SH, NRJ, and JR. DJ takes responsibility for the integrity of the data analysis. All authors have read and approved the manuscript.

\section{Funding}

The study was funded by The Odense University Hospital Research Foundation, the Region of Southern Denmark PhD Foundation, The Research Foundation between Odense University Hospital and Rigshospitalet, OPENOdense Patient data Explorative Network, The A.P. Møller Foundation for the Advancement of Medical Science, and the Danish Osteoporosis Foundation. The biobank and data management was conducted in collaboration with OPEN. The funding parties had no influence on the study design, conduct, results, or dissemination of the results.

\section{Availability of data and materials}

Data are available from the authors upon reasonable request and with permission of the Committees on Health Research Ethics, and the Data Protection Agency (2008-58-0035, 13/40496)

\section{Ethics approval and consent to participate}

This study is approved by the Committees on Health Research Ethics, the Data Protection Agency (2008-58-0035, 13/40496), and compliant with the Declaration of Helsinki. All participants gave written, informed consent prior to enrolment.

\section{Consent for publication}

Not applicable.

\section{Competing interests}

The investigators have no competing interests in the study.

\section{Author details}

${ }^{1}$ Department of Geriatric Medicine, Odense University Hospital, Odense, Denmark. Department of Clinical Research, University of Southern Denmark, Odense, Denmark. ${ }^{3}$ Department of Geriatric Medicine, Nottingham University Hospitals Trust NHS, Nottingham, UK. ${ }^{4}$ Department of Orthopaedics and Traumatology, Odense University Hospital, Odense, Denmark. ${ }^{5}$ Department of Endocrinology, Hospital South West Jutland, Esbjerg, Denmark. ${ }^{6}$ Department of Regional Health Research, University of Southern Denmark, Odense, Denmark. 'D Department of Clinical Biochemistry, Rigshospitalet, Copenhagen, Denmark. ${ }^{8}$ OPEN- Odense Patient data Explorative Network, Odense University Hospital/ University of Southern Denmark, Odense, Denmark.

Received: 1 October 2019 Accepted: 27 August 2020

Published online: 05 September 2020

\section{References}

1. Svedbom A, Hernlund E, Ivergard M, Compston J, Cooper C, Stenmark J, et al. Osteoporosis in the European Union: a compendium of countryspecific reports. Arch Osteoporos. 2013:8:137.

2. Oden A, McCloskey EV, Kanis JA, Harvey NC, Johansson H. Burden of high fracture probability worldwide: secular increases 2010-2040. Osteoporos Int. 2015;26(9):2243-8

3. Johnell $\mathrm{O}$, Kanis JA. An estimate of the worldwide prevalence and disability associated with osteoporotic fractures. Osteoporos Int. 2006;17(12):1726-33.

4. Kannegaard PN, van der Mark S, Eiken P, Abrahamsen B. Excess mortality in men compared with women following a hip fracture. National analysis of comedications, comorbidity and survival. Age Ageing. 2010;39(2):203-9.

5. Ensrud KE, Barrett-Connor EL, Schwartz A, Santora AC, Bauer DC, Suryawanshi $S$, et al. Randomized trial of effect of alendronate continuation 
versus discontinuation in women with low BMD: results from the fracture intervention trial long-term extension. J Bone Miner Res. 2004;19(8):1259-69.

6. Bone HG, Wagman RB, Brandi ML, Brown JP, Chapurlat R, Cummings SR, et al. 10 years of denosumab treatment in postmenopausal women with osteoporosis: results from the phase 3 randomised FREEDOM trial and open-label extension. Lancet Diabetes Endocrinol. 2017;5(7):513-23.

7. Neer RM, Arnaud CD, Zanchetta JR, Prince R, Gaich GA, Reginster J-Y, et al. Effect of parathyroid hormone (1-34) on fractures and Bone mineral density in postmenopausal women with osteoporosis. N Engl J Med. 2001;344(19): 1434-41.

8. Wolfson L, Judge J, Whipple R, King M. Strength is a major factor in balance, gait, and the occurrence of falls. J Gerontol A Biol Sci Med Sci. 1995;50 Spec No:64-67.

9. Sherrington C, Michaleff ZA, Fairhall N, Paul SS, Tiedemann A, Whitney J, et al. Exercise to prevent falls in older adults: an updated systematic review and meta-analysis. Br J Sports Med. 2017;51(24):1750-8.

10. Corrie H, Brooke-Wavell K, Mansfield NJ, Cowley A, Morris R, Masud T. Effects of vertical and side-alternating vibration training on fall risk factors and bone turnover in older people at risk of falls. Age Ageing. 2015;44(1):115-22.

11. Iwamoto J, Takeda T, Sato Y, Uzawa M. Effect of whole-body vibration exercise on lumbar bone mineral density, bone turnover, and chronic back pain in post-menopausal osteoporotic women treated with alendronate. Aging Clin Exp Res. 2005;17(2):157-63.

12. Roelants M, Delecluse C, Verschueren SM. Whole-body-vibration training increases knee-extension strength and speed of movement in older women. J Am Geriatr Soc. 2004;52(6):901-8.

13. Verschueren SM, Roelants M, Delecluse $C$, Swinnen $S$, Vanderschueren $D$, Boonen S. Effect of 6-month whole body vibration training on hip density, muscle strength, and postural control in postmenopausal women: a randomized controlled pilot study. J Bone Miner Res. 2004;19(3):352-9.

14. Gusi N, Raimundo A, Leal A. Low-frequency vibratory exercise reduces the risk of bone fracture more than walking: a randomized controlled trial. BMC Musculoskelet Disord. 2006;7:92.

15. Uusi-Rasi K, Kannus P, Cheng S, Sievänen H, Pasanen M, Heinonen A, et al. Effect of alendronate and exercise on bone and physical performance of postmenopausal women: a randomized controlled trial. Bone. 2003;33(1): $132-43$

16. Jepsen DB, Ryg J, Hansen S, Jorgensen NR, Gram J, Masud T. The combined effect of parathyroid hormone (1-34) and whole-body vibration exercise in the treatment of postmenopausal OSteoporosis (PaVOS study): a randomized controlled trial. Osteoporos Int. 2019.

17. Jepsen DB, Ryg J, Jorgensen NR, Hansen S, Masud T. The combined effect of parathyroid hormone (1-34) and whole-body vibration exercise in the treatment of OSteoporosis (PaVOS)- study protocol for a randomized controlled trial. Trials. 2018;19(1):186.

18. Moher D, Schulz KF, Altman DG. The CONSORT statement: revised recommendations for improving the quality of reports of parallel group randomized trials. BMC Med Res Methodol. 2001;1:2.

19. International Organization for Standardization. (ISO 13090-1:1998). Mechanica vibration and shock -- Guidance on safety aspects of tests and experiments with people -- Part 1: Exposure to whole-body mechanical vibration and repeated shock. https://www.iso.org/standard/20825.html1998.

20. Ekelund U, Sepp H, Brage S, Becker W, Jakes R, Hennings M, et al. Criterionrelated validity of the last 7-day, short form of the international physical activity questionnaire in Swedish adults. Public Health Nutr. 2006;9(2):258-65.

21. Freire AN, Guerra RO, Alvarado B, Guralnik JM, Zunzunegui MV. Validity and reliability of the short physical performance battery in two diverse older adult populations in Quebec and Brazil. J Aging Health. 2012;24(5):863-78.

22. Bassey EJ, Short AH. A new method for measuring power output in a single leg extension: feasibility, reliability and validity. Eur J Appl Physiol Occup Physiol. 1990;60(5):385-90.

23. Frederiksen H, Hjelmborg J, Mortensen J, McGue M, Vaupel JW, Christensen K. Age trajectories of grip strength: cross-sectional and longitudinal data among 8,342 Danes aged 46 to 102. Ann Epidemiol. 2006;16(7):554-62.

24. Rittweger J, Schiessl H, Felsenberg D, Runge M. Reproducibility of the jumping mechanography as a test of mechanical power output in physically competent adult and elderly subjects. J Am Geriatr Soc. 2004; 52(1):128-31.

25. Kwon S, Perera S, Pahor M, Katula JA, King AC, Groessl EJ, et al. What is a meaningful change in physical performance? Findings from a clinical trial in older adults (the LIFE-P study). J Nutr Health Aging. 2009;13(6):538-44.
26. Liphardt AM, Schipilow J, Hanley DA, Boyd SK. Bone quality in osteopenic postmenopausal women is not improved after 12 months of whole-body vibration training. Osteoporos Int. 2015;26(3):911-20.

27. Giangregorio LM, Papaioannou A, Macintyre NJ, Ashe MC, Heinonen A, Shipp K, et al. Too fit to fracture: exercise recommendations for individuals with osteoporosis or osteoporotic vertebral fracture. Osteoporos Int. 2014; 25(3):821-35.

28. Buatois $S$, Miljkovic D, Manckoundia P, Gueguen R, Miget P, Vancon G, et al. Five times sit to stand test is a predictor of recurrent falls in healthy community-living subjects aged 65 and older. J Am Geriatr Soc. 2008;56(8): $1575-7$.

29. Buatois S, Perret-Guillaume C, Gueguen R, Miget P, Vancon G, Perrin P, et al. A simple clinical scale to stratify risk of recurrent falls in communitydwelling adults aged 65 years and older. Phys Ther. 2010;90(4):550-60.

30. Jepsen DB, Thomsen K, Hansen S, Jorgensen NR, Masud T, Ryg J. Effect of whole-body vibration exercise in preventing falls and fractures: a systematic review and meta-analysis. BMJ Open. 2017;7(12):e018342.

31. Gillespie LD, Robertson MC, Gillespie WJ, Sherrington C, Gates S, Clemson LM, Lamb SE. Interventions for preventing falls in older people living in the community. Cochrane Database Syst Rev. 2012;(9):Cd007146. https://doi.org/ 10.1002/14651858.CD007146.pub3.

32. Rodrigues IB, Armstrong JJ, Adachi JD, MacDermid JC. Facilitators and barriers to exercise adherence in patients with osteopenia and osteoporosis: a systematic review. Osteoporos Int. 2017;28(3):735-45.

33. Jeffrey BA, Hannan MT, Quinn EK, Zimmerman S, Barton BA, Rubin CT, et al. Self-reported adherence with the use of a device in a clinical trial as validated by electronic monitors: the VIBES study. BMC Med Res Methodol. 2012;12:171.

34. Thorsteinsson AL, Vestergaard P, Eiken P. Compliance and persistence with treatment with parathyroid hormone for osteoporosis. Danish national register-based cohort study. Arch Osteoporos. 2015;10:35.

\section{Publisher's Note}

Springer Nature remains neutral with regard to jurisdictional claims in published maps and institutional affiliations.

\section{Ready to submit your research? Choose BMC and benefit from:}

- fast, convenient online submission

- thorough peer review by experienced researchers in your field

- rapid publication on acceptance

- support for research data, including large and complex data types

- gold Open Access which fosters wider collaboration and increased citations

- maximum visibility for your research: over $100 \mathrm{M}$ website views per year

At BMC, research is always in progress.

Learn more biomedcentral.com/submissions 